\title{
Some New Optimum Golomb Rectangles
}

\author{
James B. Shearer \\ IBM Research Division \\ T.J. Watson Research Center \\ P.O. Box 218 \\ Yorktown Heights, N.Y. 10598 \\ jbs@watson.ibm.com
}

Submitted: April 3, 1995; Accepted: June 1, 1995

Abstract: We give some new optimum Golomb rectangles found by computer search.

AMS Subject Classification. 05B99

In [2] Robinson defined a Golomb rectangle as an $N \times M$ array of ones and zeros such that the two-dimensional autocorrelation has three values: 0,1 and $K$, where $K$ is the number of ones in the array. This means that the positions of the ones in any nonzero integral translation of the rectangle will overlap with the positions of the ones in the original position of the rectangle in at most one place. Equivalently, the differences between the positions of every pair of ones in the rectangle, considered as vectors, are distinct. See also [1]. Let $G(N, M)$ be the maximum number of ones that can be present in an $N \times M$ Golomb rectangle. For example $G(2,2)=3$. Robinson defined an optimum Golomb rectangle to be one containing $G(N, M)$ ones. We prefer to add the conditions $G(N, M)>G(N-1, M)$ and $G(N, M)>G(N, M-1)$.

In table 1 we give a number of new optimum Golomb rectangles found by computer search. In most cases they are far from unique. Note there exists a $2 \times 18$ rectangle with 9 ones. The rectangle in Robinson's table $\mathrm{V}$ is $2 \times 20$ an apparent misprint.

A brief description of the computer program used to find these rectangles follows. Recall that a Golomb ruler is a set of integers $a_{1}<a_{2}<\cdots<a_{k}$ for which the $\left(\begin{array}{c}k \\ 2\end{array}\right)$ differences $\left\{a_{j}-a_{i} \mid 1 \leq i<j \leq k\right\}$ are distinct. We will use the following easy lemma.

Lemma 1: $N \times M$ Golomb rectangles with $K$ ones correspond $1-1$ with $K$ element Golomb rulers with elements chosen from the set $\{i+(2 N-1)(j-1) \mid 1 \leq i \leq N, 1 \leq$ $j \leq M\}$.

Proof: Let $\left\{\left(b_{i}, c_{i}\right) \mid 1 \leq b_{i} \leq N, 1 \leq c_{i} \leq M, 1 \leq i \leq K\right\}$ be a set of $K$ positions in a $N \times M$ rectangle. We claim this set consists of the positions of the ones in a $N \times M$ Golomb rectangle with $K$ ones iff the set $\left\{a_{i}=b_{i}+(2 N-1)\left(c_{i}-1\right) \mid 1 \leq i \leq K\right\}$, is a 
Golomb ruler with elements chosen from the set $\{i+(2 N-1)(j-1) \mid 1 \leq i \leq N, 1 \leq$ $j \leq M\}$. For suppose $\left(b_{i_{1}}, c_{i_{1}}\right)-\left(b_{i_{2}}, c_{i_{2}}\right)=\left(b_{i_{3}}, c_{i_{3}}\right)-\left(b_{i_{4}}, c_{i_{4}}\right)$. Then

$$
\begin{aligned}
a_{i_{1}}-a_{i_{2}} & =\left(b_{i_{1}}+(2 N-1)\left(c_{i_{1}}-1\right)\right)-\left(b_{i_{2}}+(2 N-1)\left(c_{i_{2}}-1\right)\right) \\
& =\left(b_{i_{1}}-b_{i_{2}}\right)+(2 N-1)\left(c_{i_{1}}-c_{i_{2}}\right) \\
& =\left(b_{i_{3}}-b_{i_{4}}\right)+(2 N-1)\left(c_{i_{3}}-c_{i_{4}}\right) \\
& =\left(b_{i_{3}}+(2 N-1)\left(c_{i_{3}}-1\right)\right)-\left(b_{i_{4}}+(2 N-1)\left(c_{i_{4}}-1\right)\right) \\
& =a_{i_{3}}-a_{i_{4}} .
\end{aligned}
$$

Conversely suppose $\left(a_{i_{1}}-a_{i_{2}}\right)=\left(a_{i_{3}}-a_{i_{4}}\right)$. Then

$$
\begin{aligned}
\left(b_{i_{1}}\right. & \left.+(2 N-1)\left(c_{i_{1}}-1\right)\right)-\left(b_{i_{2}}+(2 N-1)\left(c_{i_{2}}-1\right)\right) \\
& =\left(b_{i_{3}}+(2 N-1)\left(c_{i_{3}}-1\right)\right)-\left(b_{i_{4}}+(2 N-1)\left(c_{i_{4}}-1\right)\right) .
\end{aligned}
$$

It follows that

$$
\left(b_{i_{1}}-b_{i_{2}}\right)-\left(b_{i_{3}}-b_{i_{4}}\right)+(2 N-1)\left(\left(c_{i_{1}}-c_{i_{2}}\right)-\left(c_{i_{3}}-c_{i_{4}}\right)\right)=0 .
$$

Now $1 \leq b_{i_{1}}, b_{i_{2}}, b_{i_{3}}, b_{i_{4}} \leq N$. It follows that

$$
-(2 N-1)<\left(b_{i_{1}}-b_{i_{2}}\right)+\left(b_{i_{3}}-b_{i_{4}}\right)<(2 N-1) .
$$

Therefore we must have $\left(b_{i_{1}}-b_{i_{2}}\right)-\left(b_{i_{3}}-b_{i_{4}}\right)=0$ and $\left(c_{i_{1}}-c_{i_{2}}\right)-\left(c_{i_{3}}-c_{i_{4}}\right)=0$. Hence $\left(b_{i_{1}}, c_{i_{1}}\right)-\left(b_{i_{2}}, c_{i_{2}}\right)=\left(b_{i_{3}}, c_{i_{3}}\right)-\left(b_{i_{4}}, c_{i_{4}}\right)$. This suffices to prove the claim and the lemma.

Lemma 1 means that searches for Golomb rectangles can be performed with a modified version of the author's Golomb ruler search program (see [3]). This program performs a straightforward depth first backtrack search. It builds up a Golomb ruler by picking $a_{1}, a_{2}, \ldots, a_{k}$ in order ( $a_{j}$ being picked at level $j$ of the search tree). At each node of the search tree the program keeps track of which differences have been used (i.e. are formed by pairs of the elements which have already been picked) and of which integers can be adjoined to the current ruler without violating the distinct difference condition. The sons of a node at level $j$ are formed by setting $a_{j+1}$ to each of the elements in the level $j$ eligibility list in turn. The search tree is pruned (i.e. the program backtracks) when too few integers remain eligible to allow completion of the ruler, when not enough small differences remain unused to allow completion of the ruler or when allowed by symmetry conditions. (Symmetry conditions allow search trees to be pruned because we need only generate one member of each symmetry class of solutions. Clever use of symmetry can produce dramatic improvements in running times.) The following symmetry conditions were used in the Golomb rectangle program. Assume at least half the ones are in the left half of the rectangle (flip left and right if necessary). Assume the top half of the first column 
contains a one (clearly in an optimum rectangle the first column must contain a one, flip top and bottom if necessary). These conditions are probably not the best (in particular the second condition could assume the top half of the first column contains at least half the ones in the first column) but they were simple to implement. One can choose to search for $N \times M$ rectangles or for $M \times N$ rectangles. The main runs were done with $N \leq M$ although that is not always the best choice.

The entire program amounts to about 100 lines of VS Fortran code. As is often the case for this sort of program running times (on a 3090 IBM mainframe) increased rapidly with the problem size. For example showing $G(7,11)<14$ required 640 seconds of cpu time but showing $G(9,12)<16$ required 44000 seconds of cpu time. On average each node visited in the search tree required about 6 microseconds of cpu time.

A skeptical reader might ask why he should believe the program is correct. Checking that the rectangles found are in fact Golomb rectangles is fairly simple. An independent program checked this for the rectangles in table 1. It is possible (although tedious) to do these checks by hand. The validity of the assertion that the rectangles in table 1 are optimum (i.e. that Golomb rectangles with better parameters do not exist) is more problematic. However, the following factors give the author confidence that his search program has not missed any superior rectangles. The program successfully reproduced (with the exception noted above) the results Robinson obtained with a completely different program. The program is reasonably small (100 lines of code). Additionally, the basic algorithm and much of the code is the same as for the author's Golomb ruler program. The results obtained by the Golomb ruler program are in agreement with those obtained by other researchers using independent programs. Of course, additional checking is always possible. For example, it would be nice to do searches on $N \times M$ rectangles and on $M \times N$ rectangles as the search trees will be completely different (when $N \neq M$ ). However, as noted above this was not done for the large cases.

Table 1
$\mathrm{G}(2,18)=9 \quad 110000010000000010$
100101000000010001
$\mathrm{G}(2,29)=11 \quad 11000000100000000000010100010$
10010000000010000100000000001
$\mathrm{G}(2,35)=12 \quad 10100000000000110000000010000000100$
10010001000000000000000100001000001
$\mathrm{G}(2,43)=13 \quad 1100000000000010100000000000000000100010010$ 
THE ELECTRONIC JOURNAL OF COMBINATORICS 2 (1995) \#R12

1000001000010000000000010000000010000000001

$\mathrm{G}(2,52)=14 \quad 1100000001000000000000001000000100000000000001000101$

1001000000000100000000000000010000000000010000100000

$\mathrm{G}(2,59)=15 \quad 11000000001000000100000000000000000000001000001000000010001$

10010000000000000000001010000000000100000000000000100001000

$\mathrm{G}(3,18)=11 \quad 100100000000010100$

100000000100000001

010000011000100000

$\mathrm{G}(3,21)=12 \quad 100001000001000000011$

001000100100000000010

$\mathrm{G}(3,26)=13 \quad 10001000000010000000001101$

01000000100000000000000001

10000010000000010000100000

$\mathrm{G}(3,31)=14 \quad 1000010001000000010000001000001$

1000000000000000000010010000000

0110000000001000000000000000101

$\mathrm{G}(3,37)=15 \quad 1000010000000010000000000000000000101$

1001000000000000000000000010001000000

0010000000100000010000010000000000110

$\mathrm{G}(4,13)=11 \quad 1001000000011$

1000000000000

0100001010000

1000100000100

$\mathrm{G}(4,16)=12 \quad 1001000000001010$

1000000000000001

0000000100010000

1000011000000100

$\mathrm{G}(4,19)=13 \quad 1010000100000000100$

1000000000100000001

0000000001000001000 
THE ElECtronic JOURNAL OF COMBInATORICS 2 (1995) \#R12

0100110000000000100

$\mathrm{G}(4,23)=14 \quad 11000001000100001000000$

10000000000000000010010

00000010000000000001000

10100000000000100000001

$\mathrm{G}(4,27)=15 \quad 100000000100010100000001000$

100100000000000000000000100

000000011000000000010000001

100001000000000000000000010

$\mathrm{G}(5,11)=11$

11000000001

10000000000

00000010010

10100001000

00001000001

$\mathrm{G}(5,12)=12 \quad 110001000001$

100000000000

000000010010

100000001000

001010000001

$\mathrm{G}(5,15)=13 \quad 100001000001001$

100000000000010

000000000010001

001010000000000

100000011000000

$\mathrm{G}(5,18)=14 \quad 100000001000100001$

100000010010000000

000010000000000100

100000000000000000

010100000000000011

$\mathrm{G}(5,20)=15 \quad 10000000010000000100$

00001000100000000011

10100000000000000000

00000000000001001000

01000010000001000001 


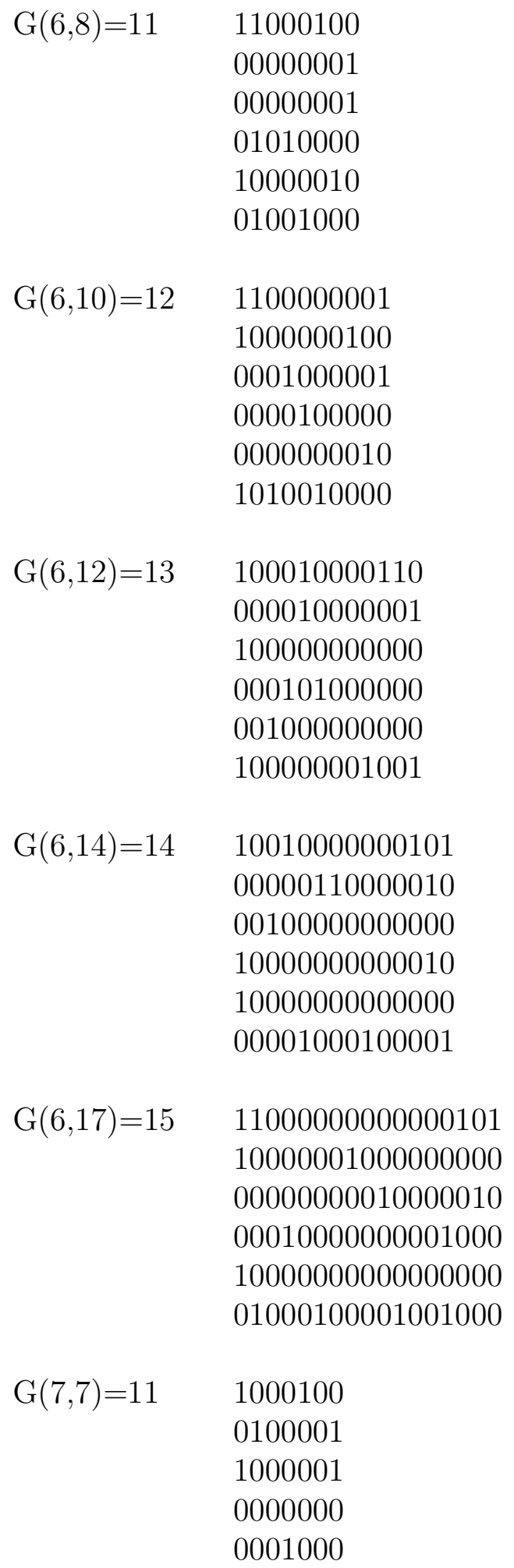


THE ELECTRONIC JOURNAL OF COMBINATORICs 2 (1995) \#R12

\begin{tabular}{|c|c|}
\hline & $\begin{array}{l}0000001 \\
0110100\end{array}$ \\
\hline $\mathrm{G}(7,9)=12$ & $\begin{array}{l}110000101 \\
100000000 \\
000100000 \\
100000000 \\
000000001 \\
010010000 \\
001000100\end{array}$ \\
\hline $\mathrm{G}(7,10)=13$ & $\begin{array}{l}1100000010 \\
0000010000 \\
0000100100 \\
1000000000 \\
0000000001 \\
1000000001 \\
0101000100\end{array}$ \\
\hline $\mathrm{G}(7,12)=14$ & $\begin{array}{l}001010000001 \\
000000001000 \\
100000000001 \\
110000000000 \\
000000000010 \\
000100100000 \\
010001000001\end{array}$ \\
\hline $\mathrm{G}(7,15)=15$ & $\begin{array}{l}100000001000011 \\
100000010001000 \\
000000000000000 \\
101000000000000 \\
000010000000001 \\
000001000000000 \\
010000000010010\end{array}$ \\
\hline $\mathrm{G}(8,8)=12$ & $\begin{array}{l}11001010 \\
10000000 \\
00000001 \\
10000000 \\
00010000 \\
00000100\end{array}$ \\
\hline
\end{tabular}


THE ElECtronic JOURNAL OF COMBInATORICS 2 (1995) \#R12

\begin{tabular}{|c|c|}
\hline & $\begin{array}{l}00100000 \\
10000001\end{array}$ \\
\hline $\mathrm{G}(8,11)=14$ & $\begin{array}{l}10000001001 \\
10001000010 \\
00000000000 \\
00001000000 \\
00000000010 \\
11000000000 \\
00000001000 \\
00100000101\end{array}$ \\
\hline $\mathrm{G}(8,13)=15$ & $\begin{array}{l}1000010001001 \\
1000000000000 \\
0000000001000 \\
0100000000000 \\
1000000000010 \\
0000000000001 \\
0000011000000 \\
0010000010100\end{array}$ \\
\hline $\mathrm{G}(9,9)=13$ & $\begin{array}{l}110000001 \\
100000000 \\
000010000 \\
100000000 \\
000000100 \\
010000010 \\
000000000 \\
100000000 \\
000101001\end{array}$ \\
\hline $\mathrm{G}(9,10)=14$ & $\begin{array}{l}1000001000 \\
1010000001 \\
0000000000 \\
1001000000 \\
0000000100 \\
0000000000 \\
0000000001 \\
1000000010 \\
0100011000\end{array}$ \\
\hline
\end{tabular}


THE ELECTRONiC JOURNAL OF COMBINATORICS 2 (1995) \#R12

$\begin{array}{ll}\mathrm{G}(9,12)=15 \quad & 110000010001 \\ & 100000000000 \\ & 000000001010 \\ & 100000000000 \\ & 000010000000 \\ & 000001000000 \\ & 000000000000 \\ & 100000000100 \\ & 000100100001 \\ \mathrm{G}(10,10)=15 & 1000010010 \\ & 0100000000 \\ & 0000000001 \\ & 1000100000 \\ & 0010000000 \\ & 0000000000 \\ & 0000000001 \\ & 0000000001 \\ & 0101000000 \\ & 1000001100\end{array}$

\section{References}

[1] Golomb, S.W. and Taylor, M., Two-dimensional synchronization patterns for minimal ambiguity, IEEE Transactions Information Theory, It-28, pp. 600-604, 1982.

[2] Robinson, J.P., Golomb Rectangles, IEEE Transactions on Information Theory, It-31, pp. 781-787, 1985.

[3] Shearer, J.B., Some New Optimum Golomb Rulers, IEEE Transactions on Information Theory, It-36, pp. 183-184, 1990. 